Editorial

\section{Early elevation of BACE1 in dementia}

Carlo Cervellati, Giuseppe Valacchi, Giovanni Zuliani

Interest in the role of Beta-secretase1 (BACE1) in Alzheimer's disease (AD) pathogenesis and pathophysiology has been remarkably growing in the last 10 years [1]. This transmembrane aspartyl protease catalyzes the rate-limiting step of the sequential cleavage of amyloid precursor protein (A $\beta \mathrm{PP})$ to form $\mathrm{A} \beta$ fragments which will then aggregate in so-called senile plaques [1].

The burst of published literature on the topic mainly stems from the potential significance of this keyenzyme of amyloidogenic pathway as pharmacological target [2]. Based on our published research we have suggested another possible clinical application of BACE1. Indeed, inspired by mounting evidence of BACE1 increase in brain, CSF, and plasma, in AD patients compared to healthy controls [1], we have started to evaluate the diagnostic performance of serum activity of the enzyme. Taking a stock of our results, from the analysis of total 817 subjects, we have found that serum BACE1 activity is around 30\% higher in patients with $\mathrm{AD}$, vascular dementia (VD), mixed dementia (AD \& VD) compared to controls $[3,4]$. Then, we moved the lens to amnestic and non-amnestic mild cognitive impairment (a- and na-MCI, respectively), with the former being more prone to convert into $\mathrm{AD}$ compared to the latter [5]. This recent investigation has revealed that serum BACE1 activity: 1) is similarly higher (around 30\%) in both aMCI and naMCIs as compared to healthy controls; 2) is higher in aMCI converting to dementia compared to stable MCI when considering patients with a less compromised cognitive status; 3 ) is not a predictor of conversion from aMCI to dementia.

The above findings can be discussed as follow:

1. The increase in BACE1 serum activity in MCI patients suggests that this enzyme is more active before the onset of overt dementia. Moreover, this abnormality appears to occur irrespectively of the possible clinical course of these patients (since it is observed in na- and a-MCI). Previous post-mortem and CSF findings on $\mathrm{MCI}$ indicate that the precocious increase in peripheral BACE1 might reflect CNS conditions [1]. More intriguingly, a recent longitudinal study has shown that plasma BACE1 level is associated with progressive axonal damage/neurodegeneration in AD critical brain region, already in cognitively and physically healthy individuals (at risk of AD) [6]. Consistently, we have also observed an association between high BACE1 and the presence of brain atrophy in aMCI [5].

These findings are not surprising, since it is well-known that $\mathrm{A} \beta$ deposition may precede $\mathrm{AD}$ clinical symptoms by approximately $15-20$ years. It is also well-established that synapse loss and dysfunction is a key feature in many neurodegenerative diseases including dementia and, in particular, AD $[1,6]$. BACE1 has the potential to be an active player in these detrimental changes, since it is able to shed several substrates, besides A $\beta P P$, implicated in, myelinization, synaptic plasticity and axonal organization. Indeed, recent approaches to identify novel BACE1 substrates, have uncovered 19\% of the 183 neuronal membrane proteins, with most of them involved in neurite outgrowth and synapsis formation, requiring this enzyme for their shedding $[1,6]$. It is thus conceivable that, undesirable BACE1 translational activation could occur precociously, and this could prime subclinical neurodegeneration.

2. The selective increase of serum BACE1 activity in converter aMCI with a higher MMSE might suggest that this could be a risk factor for dementia in those patients where the cognitive decline has not overtly taken place. It is tempting to speculate, that the contribution of BACE1 in the clinical trajectory to dementia is tangible only when other pathogenic players have not yet become predominant. Indeed, while it is widely recognized that $A \beta$ formation is the initial event in $\mathrm{AD}$, it has also become increasingly apparent that secondary abnormalities such as tau and neuroinflammation might play a major role in the subsequent neurodegeneration [7]. In other words, for MCIs with pronounced cognitive symptoms, the impact of tauopathy and inflammation may be dominant and mask the contribution of BACE1 in the progression to the disease. Apparently, the results we obtained by Cox model (median follow up of 32 months) do not support the use of serum BACE1 activity as prediction biomarker for $\mathrm{AD}$ and dementia in general [5]. However, it must be underscored that our final sample was definitely underpowered for this kind of analysis. Given the above mentioned results and the clear trend of Cox curve, we believe that a larger number of MCI with good cognitive performance could give different results. 
In summary, BACE1 up-regulation might be one of the first abnormal event in AD (and VD). Within this long preclinical phase of the disease, which lasts years before the diagnosis of MCI state, BACE1 may be the keyplayer of the initial, and still asymptomatic, neuropathological changes. In this time frame, the evaluation of its serum activity might have clinical relevance as early biomarker, mostly for screening subjects to enroll in clinical trials. The time when $A \beta$ accumulation is the prominent alteration, and the specific weight of other pathogenic actors is still relatively low, BACE1 could also be candidate therapeutical target. As recently suggested by Panza et al.[2], large random clinical trials with BACE1 inhibitors have failed (for lack of benefit or the occurrence of adverse effects) most likely because they were administered on patients experiencing the irreversible phase of the disease (mild-to-moderate AD). Indeed, in these treated patients the significant decrease in cerebrospinal fluid $\mathrm{A} \beta$ (but not in amyloidPET), is not accompanied by cognitive benefit [2]. This could mean that BACE1-mediated formation of $A \beta$ has lost its "primacy", and other pathogenic players could have taken over the lead.

\section{REFERENCES}

1. Hampel H, et al. Biol Psychiatry. 2021; 89:745-56. https://doi.org/10.1016/i.biopsych.2020.02.001 PMID:32223911

2. Panza F, et al. Expert Rev Neurother. 2018; 18:84757. https://doi.org/10.1080/14737175.2018.1531706 PMID: $\underline{30277096}$

3. Zuliani G, et al. Sci Rep. 2020; 10:14980. https://doi.org/10.1038/s41598-020-72168-3 PMID:32917964

4. Cervellati C, et al. Geroscience. 2020; 42:159-67. https://doi.org/10.1007/s11357-019-00127-6 PMID: $\underline{31745860}$

5. Zuliani G, et al. J Neurochem. 2021; 159:629-37. https://doi.org/10.1111/inc.15513 PMID:34534363

6. Vergallo A, et al. Alzheimers Dement. 2021; 17:62940. https://doi.org/10.1002/alz.12228 PMID: 33527718

7. Cervellati C, et al. Antioxid Redox Signal. 2020; 33:191-210. https://doi.org/10.1089/ars.2020.8076 PMID: $\underline{32143546}$
Carlo Cervellati: Department of Translational Medicine and for Romagna, University of Ferrara, Ferrara 44121, Italy

Correspondence: Carlo Cervellati

Email: crvcrl@unife.it

Keywords: Alzheimer's disease, amyloid-6, BACE1, mild cognitive impairment

Acknowledgements: The authors would like to thank Dr. Valentina Rosta and Dr. Alessandro Trentini for their excellent and essential contribution to the studies which inspired this work

Copyright: (C) 2021 Cervellati et al. This is an open access article distributed under the terms of the Creative Commons Attribution License (CC BY 3.0), which permits unrestricted use, distribution, and reproduction in any medium, provided the original author and source are credited

Received: November 11, 2021

Published: November 29, 2021 